\title{
Drowsiness Detection using CNN
}

\author{
Sheela. $\mathrm{S}^{\mathrm{a}, 1}$, Jothika. D. $\mathrm{E}^{\mathrm{b}}$, Swarnapriya. $\mathrm{N}^{\mathrm{b}}$, Vishali. B. $\mathrm{R}^{\mathrm{b}}$ \\ ${ }^{a}$ Assistant Professor, Dept of ECE, Rajalakshmi Institute of Technology, India \\ ${ }^{b}$ Dept of ECE, Rajalakshmi Institute of Technology, India
}

\begin{abstract}
Driver drowsiness has become one of the leading causes of car accidents in recent years resulting in serious physical injuries, fatalities and substantial financial losses. According to statistics, are liable driver drowsiness detection system is needed to warn the driver before a collision occurs. Behavioural, physiological and vehicle based measures are used by researchers to assess driver drowsiness. A thorough examination of these measures will shed light on the current systems, their problems, and the improvements that must be made in order to create a reliable system. By doings other measures will reveal existing systems, their flaws and the changes that must be made in order to produce a credible system.
\end{abstract}

Keywords: Drowsiness, Neural Network, Image processing.

\section{Introduction}

According to available statistics, each year people die on the road is nearly 1.3 million and around 50 million peoples are suffering from non-fatal injuries as a result of traffic accidents. At the wheel if a driver falls asleep, the car loses its control and crashes into another vehicle or stationary objects. The driver's level of drowsiness should be monitored to stop these fatal accidents. The following tools have been widely used to track drowsiness:

1)Vehicle based measures: Deviations from lanes, steering wheel rotation, accelerator pedal pressure, and other factors are continuously tracked, and any change that exceeds a predetermined level signals indicates that the driver is drowsy.

2) Behavioural measures: A camera tracks the driver's actions, such as eye closing, eye twitch, head posture, and yawning, and if any of these drowsiness signs are observed the driver is alerted. 3)Physiological measures: Many studies have looked into the relationship between physiological signals (ECG,EMG,EoG and EEG) and driver drowsiness. One of the important safety features is drowsiness detection that can avoid accidents caused by driver's drowsiness.

The aim of this study is to detect and alert the person when eyes are not opened for a particular duration. When drowsiness is detected, this system will notify the driver.

\footnotetext{
${ }^{1}$ Sheela.S,AssistantProfessor,RajalakshmiInstituteofTechnology,Chennai,India.

Emailid: ssheela97@gmail.com
} 


\section{Methodology}

Driver exhaustion and drowsiness are significant contributors to various automobile accidents. In the field of accident prevention systems, designing and maintaining technology that can effectively identify or avoid drowsiness at the wheel and warn the driver before a collision is a major challenge. We use OpenCV to take images from a webcam and these images given to a deep learning algorithm that can tell whether someone's eyes are closed or opened. In this case, we are looking for the persons face and eyes.

Step1: Image is taken as input from camera.

We'll use a camera to capture photographs as input. But, in order to gain access to the webcam, we created an endless loop that captures each frame. We employ the cv2 method given by OpenCV. VideoCapture(0) (cap) is used to access the camera and capture the object. With cap.read(), each frame is read, and then image is saved in a variable.

Step 2:Create a ROI by detecting a face in the picture.

To segment the face in the captured image, we first converted it to gray scale because, the OpenCV object detection algorithm only accepts grayscale images as input. To detect the objects, we don't need colour detail. We use the Haar cascade classifier to detect the face. The classifier face $=$ cv2.Cas is set with this section. for (x,y,w,h)infaces, we use cv2.rectangle(frame, $(x, y),(x+w, y+h),(100,100,100), 1$

Step 3: Use the ROI to find the eyes and feed them to the classifier.

The technique for detecting eyes is the same as for detecting ears. Cascade classifier is used in left and right eyes.

Then, use left eye=leye.detectMultiScale(gray) to detect the eyes. We extracted only the details of eyes from the captured image. This can be done by first removingthe eye's boundary box and then using this code to remove the eye image from the picture.

$$
\text { 1_eye }=\text { frame }[y: y+h, x: x+w]
$$

This information is given to $\mathrm{CNN}$, which decides whether the eyes are closed or not. The right eye also detected in the above manner.

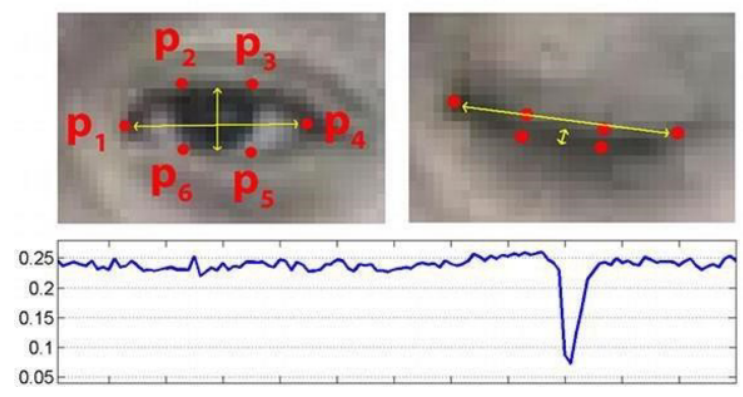

Figure1: Detection of Eyes using OpenCV 
Step 4 - The classifier will determine whether or not the eyes are open.

The eye status is predicted using a CNN classifier to feed the image into the model, since the model requires the proper measurements to begin with. We begin by converting the colour picture to grayscale.

$$
\text { r_eye=cv2.cvtColor(r_eye,cv2.COLOR_BGR2GRAY). }
$$

Then, since the model is trained on images with a resolution of $24 * 24$ pixels, We resize the image to $24 * 24$ pixels.

cv2.resize (r_eye, $(24,24))$.

For better convergence, the date is normalized.

$$
\text { r_eye }=\text { r_eye } / 255
$$

The model is loaded using model=load_model(,,models/cnnCat2.h5 $5^{\text {ee }}$ )

Now, each eye is predicted with the proposed model.

lpred=model.predict_classes(1_eye)

If $\operatorname{lpred}[0]=1$, it means that eyes are open, if $\operatorname{lpred}[0]=0$ then, it means that eyes are closed.

Step 5: Score Calculation.

The score is essentially a number that we'll use to figure out how long the individual has been closed-eyed. As a consequence, if both eyes are closed, we will begin to raise the score, but if both eyes are open, we will decrease the score. We're using the cv2.putText() function to draw the result on the screen, which displays the status of the driver or a person.

$$
\text { cv2.putTxt(frame,“Open”,(10,height20), font,1,(255,255,255),1,cv2.LINE_AA ) }
$$

A criterion is established, for example, if the score exceeds 15 , it indicates that the person's eyes have been closed for an extended amount of time. Then the alarm turned on.

Step 6: To run the file

You can either open the command prompt or navigate to the directory containing our key file

"drowsiness detection.py" as shown on the picture above by using the command below: Python

"drowsiness detection.py" 


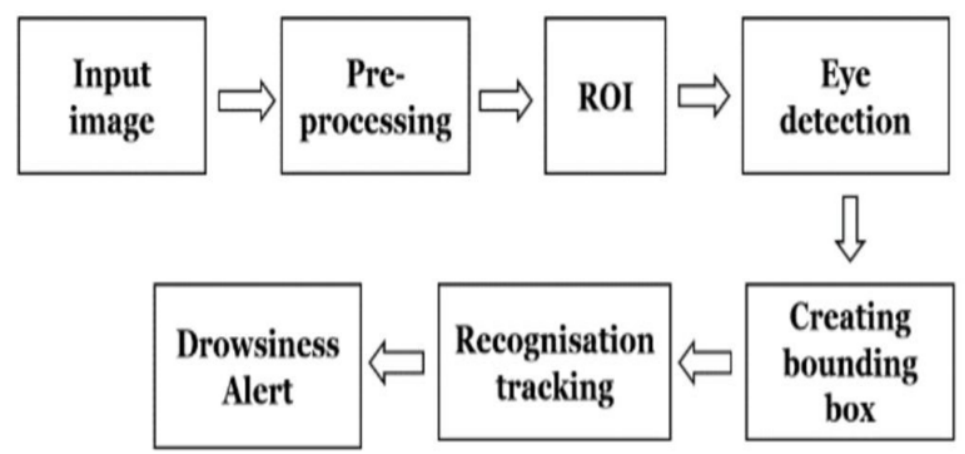

Figure 2: Block Diagram for Drowsiness Detection Using CNN

\section{Results and Discussions}

The first image output shows the normal state of the person without any signs of drowsiness so the score value is 0 . As for the second image the score value is beyond 15 so the detection system alerts the person by a sound alarm creating a bounding box.

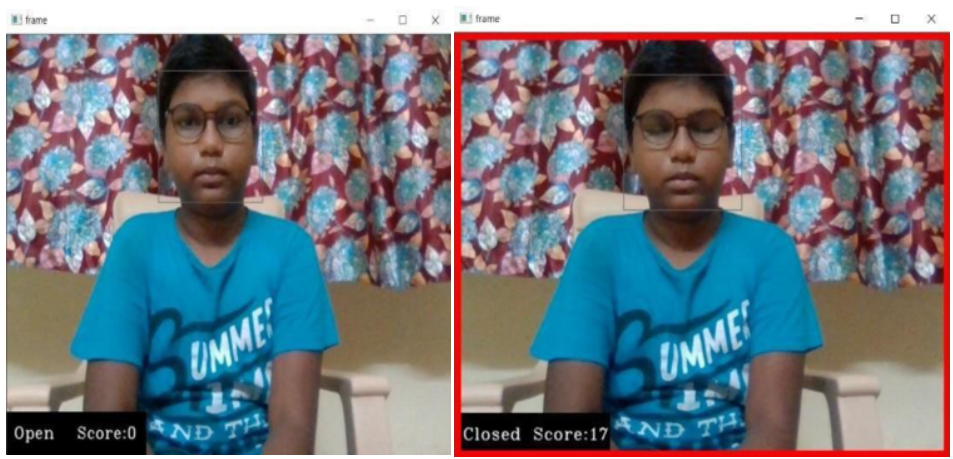

Figure 3: Drowsiness Detection

\section{Conclusion}

The Drowsiness Detection System, which is based on the driver's eye closure, can discern between normal eye twitch and drowsiness, as well as detect drowsiness when driving. The suggested scheme will help deter injuries caused by drowsy driving. Using a Haar cascade classifier, OpenCV was used to detect faces and eyes, and then a CNN model to predict the status. A alert signal is provided when the eyes are closed for an extended amount of time. Continuous eye closures are used to assess the driver's alertness level. For the future work, this detection system can be made into hardware with advanced features. 


\section{References}

[1] Ann Williamson and Tim Chamberlain. Review of on-road driver fatigue monitoring devices. NSW Injury Risk Management Research Centre, University of New South Wales, , July2013.

[2] C. Hentschel, T. P. Wiradarma, and H. Sack, Fine tuning CNNS with scarce training data-adapting imagenet to art epoch classification, in Proceedings of the IEEE International Conference on Image Processing (ICIP), Phoenix, AZ, USA, September 2016.

[3] K. He, X. Zhang, S. Ren, and J. Sun, Deep residual learning for image recognition, in Proceedings of the IEEE Conference on Computer Vision and Pattern Recognition, Las Vegas, NV, USA, June 2016.

[4] ZuopengZhao,LanZhang,Hualin Yan, Yi Xu, and Zhongxin Zhang, Driver Fatigue Detection Based on Convolutional Neural Networks Using EM -CNN, 2020.

[5] MiluPrince,NehaSanthosh,NehaSanthosh, Reshma Sudarsan \& Ms.AnjusreeV.K,Rajagiri School of Engineering And Technology - Eye Movement Classification Using CNN.

[6] AnjithGeorge and AurobindaRoutrayDept. of Electrical Engg., IIT Kharagpur Kharagpur, 721302, India - "Real-time Eye Gaze Direction Classification Using Convolutional NeuralNetwork".

[7] HaoxiangLi ,ZheLin, XiaohuiShen , Jonathan Brandt, Gang HuStevensInstitute of Technology Hoboken, NJ 07030- A Convolutional Neural Network Cascade for FaceDetection.

[8] Venkata Rami Reddy Chirra,Srinivasulu Reddy Uyyala\& Venkata Krishna Kishore Kolli, Department of Computer Applications, National Institute of Technology, Tiruchirappalli 620015, India - “Deep CNN: A Machine Learning Approach for Driver Drowsiness Detection Based on Eye State

[9] V.D.Ambeth Kumar et.al., , "Performance Improvement Using an Automation System for Segmentation of Multiple Parametric Features Based on Human Footprint" for the Journal of Electrical Engineering \& Technology , vol. 10, no. 4, pp.1815-1821 , 2015. [http://dx.doi.org/10.5370/JEET.2015.10.4.1815]

[10] Ambeth Kumar.V.D et.al. .A Survey on Face Recognition in Video Surveillance. Lecturer Notes on Computational and Mechanism, Vol. 30, pp: 699-708, 2019

[11] Ambeth Kumar.V.D .Precautionary measures for accidents due to mobile phone using IOT.Clinical eHealth, Volume 1, Issue 1, March 2018, Pages 30-35.

[12] Ambeth Kumar.V.D et,al. Enhancement in Footprint Image using Diverse Filtering Technique. Procedia Engineering journal, Volume 8, No.12, 1072-1080, 2012. [doi:10.1016/j.proeng.2012.01.965]

[13] Nanagasabapathy.K et.al, Validation system using smartphone luminescence. IEEE International Conference on Intelligent Computing, Instrumentation and Control Technologies (ICICICT), Pages: 235 - 239, 6-7 July 2017, Kannur, India

[14] Aravindh.B et.al, A novel graphical authentication system for secure banking systems. IEEE International Conference on Smart Technologies and Management for Computing, Communication, Controls, Energy and Materials, Pages: 177 - 183, 2-4 Aug. 2017, Vel Tech University, Chennai, India

[15] S.V. Ruphitha et.al, Management of Major Postpartum Haemorrhage by using Zigbee protocol - A Review .2021 6th International Conference on Inventive Computation Technologies (ICICT) (DOI: 10.1109/ICICT50816.2021.9358757 )

[16] Indhumathi.M et.al .Healthcare Management of Major Cardiovascular Disease-A review.2021 6th International Conference on Inventive Computation Technologies (ICICT), (DOI: 10.1109/ICICT50816.2021.9358519) 